\title{
Syndecan-4 Signaling Is Required for Exercise-Induced Cardiac Hypertrophy
}

\author{
Jun Xie, ${ }^{1}$ Guixin He, ${ }^{1,3}$ Qinhua Chen, ${ }^{1}$ Jiayin Sun, ${ }^{2}$ Qin Dai, ${ }^{1}$ Jianrong $L u^{1}$, Guannan Li, ${ }^{1}$ Han Wu, ${ }^{1}$ Ran Li $^{1}$ \\ Jianzhou Chen, ${ }^{1}$ Wei $\mathrm{Xu},{ }^{1}$ and Biao $\mathrm{Xu}{ }^{1}$
}

${ }^{1}$ Department of Cardiology, Drum Tower Hospital, Nanjing University Medical School, Nanjing, China; ${ }^{2}$ Department of VIP, Beijing Anzhen Hospital, Capital Medical University, Beijing, China; and ${ }^{3}$ Department of Cardiology, the First Affiliated Hospital of Guangxi University of Chinese Medicine, Nanning, Guangxi, China

\begin{abstract}
Cardiac hypertrophy can be broadly classified as either physiological or pathological. Physiological stimuli such as exercise cause adaptive cardiac hypertrophy and normal heart function. Pathological stimuli including hypertension and aortic valvular stenosis cause maladaptive cardiac remodeling and ultimately heart failure. Syndecan-4 (synd4) is a transmembrane proteoglycan identified as being involved in cardiac adaptation after injury, but whether it takes part in physiological cardiac hypertrophy is unclear. We observed upregulation of synd 4 in exercise-induced hypertrophic myocardium. To evaluate the role of synd 4 in the physiological form of cardiac hypertrophy, mice lacking synd4 (synd $4^{-1}$ ) were exercised by swimming for 4 wks. Ultrasonic cardiogram (UCG) and

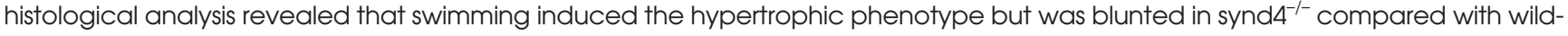
type (WT) mice. The swimming-induced activation of Akt, a key molecule in physiological hypertrophy was also more decreased than in WT controls. In cultured cardiomyocytes, synd4 overexpression could induce cell enlargement, protein synthesis and distinct physiological molecular alternation. Akt activation also was observed in synd4-overexpressed cardiomyocytes. Furthermore, inhibition of protein kinase C (PKC) prevented the synd4-induced hypertrophic phenotype and Akt phosphorylation. This study identified an essential role of synd 4 in mediation of physiological cardiac hypertrophy.
\end{abstract}

Online address: http://www.molmed.org

doi: $10.2119 / \mathrm{molmed} .2015 .00026$

\section{INTRODUCTION}

Regular exercise has many beneficial effects, including direct adaptations of the heart that result in physiological hypertrophy, enhanced contraction and accelerated relaxation (1). By contrast, pathological hypertrophy occurs in response to cardiac injury and results in the loss of cardiomyocytes and heart failure. Physiological cardiac hypertrophy has been shown to decrease the risk of the development of pathological cardiac hypertrophy and cardiac enlargement (2), improve the quality of life and decrease mortality for heart failure patients (3). Hence, the knowledge about the mechanism in physiological cardiac hypertrophy is essential for the development of novel and viable therapeutic intervention strategies for patients with cardiovascular disease and heart failure.

The phenotype of physiological hypertrophy is distinct from pathological cardiac hypertrophy at the cardiomyocyte level. For example, ventricular

Address correspondence to Biao Xu and Wie Xu, Department of Cardiology, Drum Tower Hospital, Nanjing University Medical School, Zhongyang Road, Nanjing, 210008, China. Phone: +86-25-68182812; Fax: +86-25-68182812; E-mail: xubiao@medmail.com.cn and xuwei2003@medmail.com.cn.

Submitted February 1, 2015; Accepted for publication January 20, 2016; Published Online (www.molmed.org) January 21, 2016.

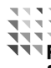

Feinstein Institute
for Medical Research Northwell Health

cardiomyocytes of exercise-trained mice exhibit increased size, enhanced lipid metabolism and greater contraction due to increased contractile proteins (4). The identification of distinct signaling in the development of these two forms of hypertrophy has proven difficult. Only a few signaling pathway are demonstrated to act as distinct signaling cascades to regulate physiological or pathological growth of the heart, for example phosphoinositide 3-kinase p $110 \alpha$ (PI3K $[\mathrm{p} 110 \alpha]$ ) for physiological cardiac hypertrophy (5) or calcineurin-nuclear factor of activated T-cells (NFAT) signaling for pathological cardiac hypertrophy (6). The roles of a large amount of molecules in these two forms of cardiac hypertrophy are ambiguous. It is possible that some molecule could initiate different downstream signaling to mediate different phenotypes associated with physiological and pathological hypertrophy, such as L-type calcium 
channel and mammalian target of rapamycin complex 1 (mTORC1) $(7,8)$.

Syndecan 4 (synd4) is a transmembrane proteoglycan that connects extracellular matrix proteins to the cardiomyocyte cytoskeleton (9) and has been localized in costameres and Z-discs (10), potentially important sites for signal transduction across the membrane. Moreover, synd 4 could initiate its downstream pathway via protein kinase $\mathrm{C} \alpha(\mathrm{PKC} \alpha)(11)$, which seems to be required for cardiac adaptations to several stimulations (12). Our previous report (13) and works of other authors $(14,15)$ have indicated that synd 4 takes part in cardiac hypertrophy as an adaptation in the myocardium infarction model and the transverse aortic constriction (TAC) model. Calcineurin-NFAT pathway is identified as downstream signaling of synd 4 in pathological cardiac hypertrophy. Meanwhile, previous studies show synd 4 could activate the Akt pathway in endothelial cell $(16,17)$, one of important pro-physiological hypertrophy kinases (18). Whether synd 4 can also initiate the Akt pathway in cardiomyocytes and associate with cardiac hypertrophy stimulated by physiological stimulation needs further exploration.

In this study, we use synd 4 knockout mice to identify the essential role of synd 4 in physiological cardiac hypertrophy and demonstrate that synd4 could induce physiological cardiac hypertrophy via PKC and Akt pathway.

\section{MATERIALS AND METHODS}

Detailed materials and methods are available in the online-only Data Supplement. All experimental procedures and research were performed in accordance with ethical standards formulated in the Declaration of Helsinki of 1975 (revised 1983) and the guidelines in the Guide for the Care and Use of Laboratory Animals published by the National Institutes of Health (National Institutes of Health publication no. 85-23, revised 1985) and were approved by the
Institutional Ethics Committee of Nanjing Drum Tower Hospital (Approval No. 201325).

\section{Experimental Animals}

Synd $4^{-/-}$mice, backcrossed more than 10 generations onto the C57BL/ 6 background, were obtained from the Center for Animal Resources and Development at Kumamoto University. Twelve-week-old male Syn $4^{-/}$mice and age-matched male wild-type (WT) mice on a C57BL/ 6 background were used in the present study. Four-monthold Sprague-Dawley (SD) rats were obtained from the Center for Animal Resources and Development at Nanjing University. All animals were kept on a 12-h light-dark cycle and food and water were provided ad libitum.

\section{Experimental Protocols}

A swimming protocol mainly described by Masayuki Taniike et al. was used (19). Mice and rats were exercised by swimming. Mice and rats were adapted to the swimming program by beginning with $10 \mathrm{~min}$ sessions two times a day separated by $4 \mathrm{~h}$. These sessions were increased by 10 min every day until reaching $90 \mathrm{~min} /$ session. The program was completed in 4 wks. During each session, mice and rats were monitored for inability to sustain the exercise and/or sudden death. Mice and rats were euthanized by cervical dislocation under ether after $4 \mathrm{wks}$ of swimming and one week of rest, and histological analysis was done. Detailed description of ultrasonic cardiogram and histological analysis are provided in the online-only Data Supplement.

\section{Primary Culture of Neonatal Mice Ventricular Cardiomyocytes (NMVCs)}

We obtained ventricles from 1-to-3-d-old mice and isolated cardiomyocytes through digestion with trypsin-ethylenediaminetetraacetic acid (trypsin-EDTA) and type 2 collagenase as described previously (20). The NMVCs were identified by sarcomeric actin staining (Supplementary Figure 1). Thereafter, neonatal mice cardiomyocytes (NMCMs) were subjected to immunofluorescence, quantitative real-time PCR analyses or Western blots. Detailed descriptions of NMVCs culture is provided in the online-only Data Supplement.

\section{Construction and Preparation of Recombinant Adenoviruses}

Recombinant adenoviruses for gene delivery were prepared using the pAd-Easy system, as described previously (13). A cDNA corresponding to the coding region of human syndecan-4 (NM_002999) was subcloned into pShuttle-CMV. The adenovirus was constructed and named Ad-synd4. The synd 4 expression was verified by Western blotting analysis. Similarly, an adenovirus that contained $\mathrm{Lacz}$ gene following the CMV promoter was prepared. This virus, named Ad-Lacz, was used as a control in parallel studies.

\section{mRNA Quantification}

Total RNA was extracted using TRIzol (Invitrogen). cDNA was produced by using PrimeScript RT reagent Kit (TaKaRa). mRNA expression was analyzed with quantitative real-time RT-PCR using a SYBR Premix Ex Taq System (TaKaRa). Amplification and detection were performed using 7500HT Fast Real-Time PCR system (Applied Biosystems). The primers used in this study are provided in the online-only Data Supplement.

\section{Western Blot Analysis}

Protein loading was confirmed by Coomassie blue staining. $\beta$-actin and histone $\mathrm{H} 3$ were used as loading controls. To determine NFAT3 cellular localization, nuclear and cytoplasmic proteins were isolated from heart tissues using a cell fractionation kit (Keygen). Primary antibodies used were anti-synd4 (1:500, Lifespan Biosciences), anti-PPAR $\alpha$ (1:500, Bioworld Technology), anti-ANF (1:500, BD), anti-Akt (1:1,000, Cell Signaling Technology), 
A

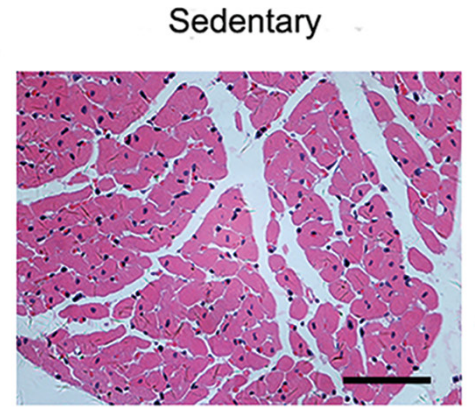

\section{B}
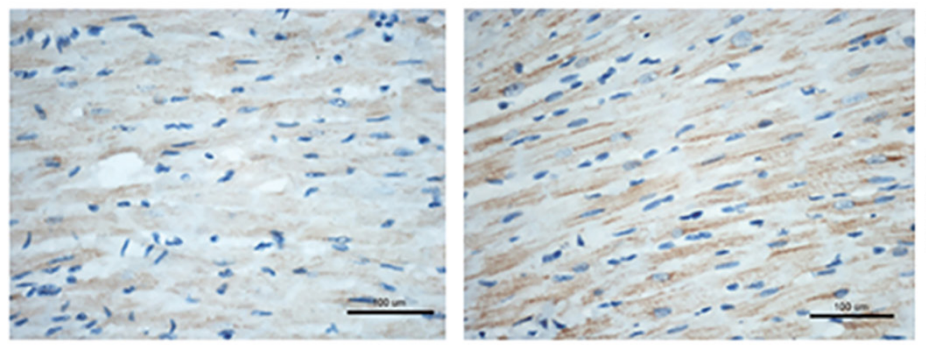

Exercise
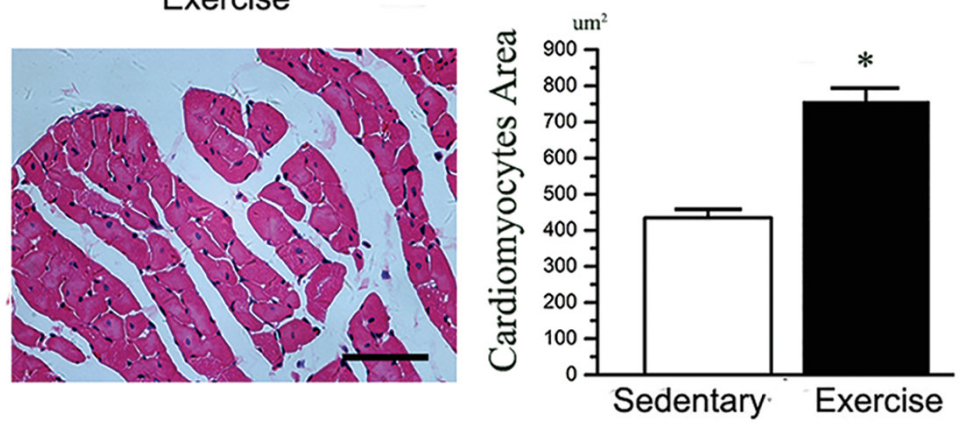
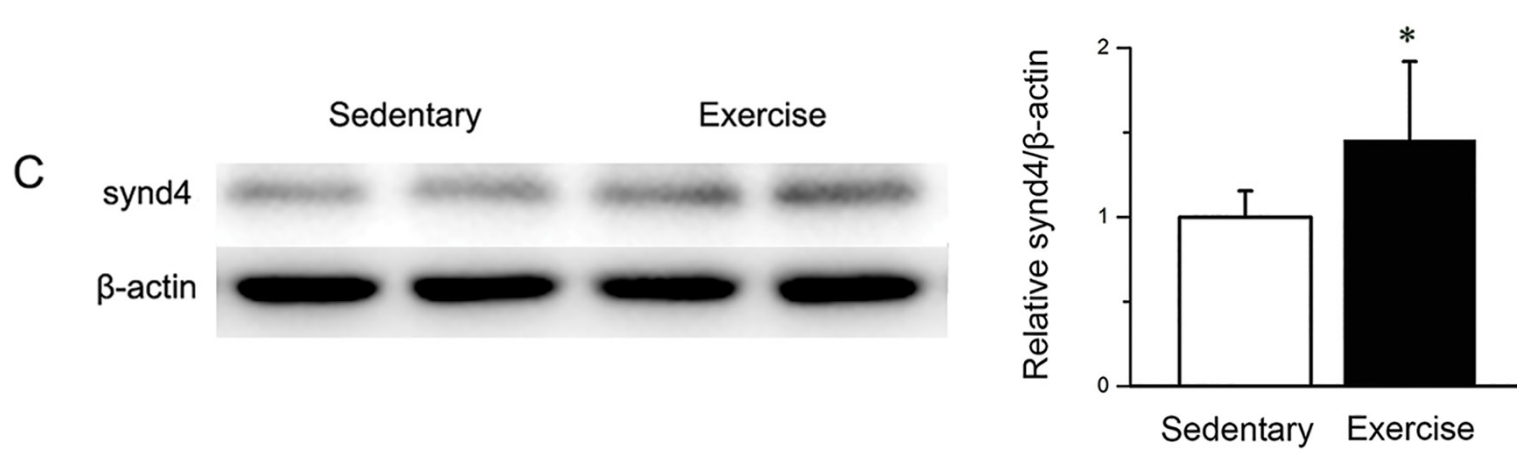

Figure 1. Aerobic interval swimming training was used to induce physiological cardiac hypertrophy in rats, and synd4 was detected by immunohistochemistry assay and Western blot. (A) Histology analysis showed increases in cardiomyocyte surface area in Ex-rats compared with Sed-rats. ${ }^{*} P<0.05$, Ex-rats versus Sed-rats; $N=6$; scale bar $=100 \mu \mathrm{m}$. (B) Immunohistochemistry assay showed high intensity of synd4 expression in the cardiomyocytes of Ex-rats. ${ }^{*} P<0.05$, Ex-rats versus Sed-rats; $N=6$; scale bar $=100 \mu \mathrm{m}$. (C) Western blot showed increases of synd 4 expression in myocardium after exercise. Data are shown as a ratio to $\beta$-actin and are expressed as a fold-increase when compared with Sed-rats. ${ }^{*} P<0.05$, Ex-rats versus Sed-rats; $N=6$; synd4: syndecan-4.

anti-p-Akt (308) (1:1,000, Cell Signaling Technology), anti-p-Akt (473) (1:1,000, Cell Signaling Technology), anti-calcineurin (1:2,000, Abcam), anti-NFAT3 (1:1,000, Cell Signaling Technology), anti-histone H3 (1:500, Bioworld Technology) and anti- $\beta$ actin (1:1,000, Bioworld Technology).

\section{Statistical Analysis}

Data were expressed as mean \pm SEM. All data analysis was performed with the use of SPSS 16.0 software. Statistical significance was defined as
$P<0.05$ (two-tailed). Whether the distribution of the continuous variables was normal was assessed with the Shapiro-Wilk test. Comparison of parameters among the three groups was performed by analysis of variance (ANOVA) or Kruskal-Wallis tests for normally and nonnormally distributed data, respectively. Gene expression was analyzed by the $\Delta \Delta \mathrm{Ct}$ method using online software from SABiosciences. Values of $P<0.05$ were considered statistically significant.
All supplementary materials are available online at www.molmed.org.

\section{RESULTS}

\section{Exercise Induced Cardiac Adaptation and Increased Synd4 Expression in Myocardium}

Following a 4-wk highly intense aerobic interval swimming training, exercise-trained rats (Ex-rat) had increased physiological cardiac hypertrophy as compared with sedentary rat 
Table 1. Echocardiographic parameters of rats after swimming training.

\begin{tabular}{lcc}
\hline & Ex-rats $(n=7)$ & Sed-rats $(n=7)$ \\
\hline IVSD $/ \mathrm{mm}$ & $1.84+0.021$ & $2.264+0.027^{a}$ \\
$\mathrm{LVPWD} / \mathrm{mm}$ & $1.797+0.027$ & $2.307+0.03^{a}$ \\
$\mathrm{LVEDD} / \mathrm{mm}$ & $5.97+0.031$ & $6.05+0.046$ \\
$\mathrm{LVESD} / \mathrm{mm}$ & $3.103+0.029$ & $3.094+0.014$ \\
$\mathrm{FS} / \%$ & $48.02+0.48$ & $48.84+0.32$ \\
$\mathrm{EF} / \%$ & $72.97+0.5$ & $73.82+0.33$ \\
\hline
\end{tabular}

${ }^{a} P<0.05$.

without swimming training (Sed-rat). Echocardiography was used to assess heart dimensions and function in rats. As shown in Table 1, the hearts from Ex-rats displayed a significant increase in interventricular septal thickness in diastole (IVSD) and lower ventricular posterior wall thickness in diastole (LVPSD), compared with Sed-rats.

To assess whether the increased myocardial wall thickness in the Ex-rats represented increases in cardiomyocyte, cell surface area was measured in whole heart cross-sections (Figure 1A). The cardiomyocyte cross-sectional surface areas were clearly larger in the Ex-rats compared with the Sed-rats. These data indicated that the training protocol had achieved a ventricular hypertrophy. We further explored the synd 4 expression in the myocardium of the rats. In immunohistochemistry assay, a high intensity of synd 4 expression in the cardiomyocytes of Ex-rats was observed (Figure 1B). The result of Western blot test regarding synd 4 expression was similar (Figure 1C).

\section{Hypertrophic Phenotype was Blunted in Synd4 ${ }^{-/-}$Mice after Exercise}

We observed upregulation of synd 4 and cardiac hypertrophy in exercise-trained animals. To explore whether synd 4 is required in exercise-induced cardiac hypertrophy, synd 4 knockout mice were used in our training protocol. UCG was used to compare cardiac structure and function between transgenic and WT mice. The

Table 2. Echocardiographic parameters of mice at basal level and after swimming training.

\begin{tabular}{lccccc}
\hline & \multicolumn{2}{c}{ At basal level } & & \multicolumn{2}{c}{ After swimming } \\
\cline { 2 - 3 } \cline { 6 - 6 } & WT $(n=12)$ & Synd4 $^{-/-}(n=12)$ & & WT $(n=12)$ & Synd $^{-/-}(n=12)$ \\
\hline IVSD $/ \mathrm{mm}$ & $0.648+0.0074$ & $0.677+0.0139$ & & $0.804+0.009$ & $0.698+0.0161^{a}$ \\
$\mathrm{LVPWD} / \mathrm{mm}$ & $0.637+0.0112$ & $0.677+0.0109$ & & $0.831+0.0261$ & $0.70+0.0228^{a}$ \\
$\mathrm{LVEDD} / \mathrm{mm}$ & $3.16+0.12$ & $3.17+0.06$ & & $3.35+0.09$ & $3.46+0.12$ \\
$\mathrm{LVESD} / \mathrm{mm}$ & $1.91+0.05$ & $2.05+0.09$ & & $2.20+0.08$ & $2.22+0.11$ \\
$\mathrm{EF} / \%$ & $62.82+3.02$ & $58.37+2.29$ & & $56.98+1.63$ & $58.48+2.76$ \\
$\mathrm{FS} / \%$ & $38.03+1.21$ & $38.01+1.34$ & & $37.97+0.83$ & $35.74+2.13$ \\
\hline
\end{tabular}

${ }^{a} P<0.05$ versus corresponding control.

synd $4^{-/-}$mice with a C57Bl6/J mice background appeared with normal parameters of the left ventricle and had a normal global cardiac function before the training protocol (Table 2), as presented in a previous study (21). After swimming for $4 \mathrm{wks}$, the IVSD and left ventricular posterior wall thickness in diastole (LVPWD) in WT mice were increased significantly as compared with those in synd $4^{-/-}$mice. The diameter of the left ventricle, as indicated by left ventricular end-systolic diameter (LVESD) and left ventricular enddiastolic diameter (LVEDD), showed no difference between WT and synd $4^{-/-}$ mice, though the diameters in both groups were slightly increased after training. Cardiac function, as indicated by fractional shortening (FS) and ejection fraction (EF), was also no different between WT and synd $4^{-/-}$mice after exercise (Table 2).

In the histological assay, cardiac hypertrophy was assessed by measuring the ratio of heart weight to body weight (HW/BW). HW / BW increased significantly in WT mice compared with synd $4^{-/-}$mice after the swimming program (Figures 2A-C). The cardiomyocyte cross-sectional area also increased significantly in WT as compared with synd $4^{-/-}$mice (Figure 2D). The level of fibrosis and apoptosis in the ventricular wall were normal in both groups (Figures 2E,F). These results indicated that WT mice developed a more profound cardiac hypertrophy than synd $4^{-/-}$mice after undergoing 4 wks of exercise.

\section{Synd4 Induced Physiological Hypertrophic Phenotype in Cardiomyocytes}

In the animal study, we found synd 4 is essential for cardiac hypertrophy under exercise. Next, we explored whether synd 4 could initiate physiological hypertrophy in cardiomyocytes. Ad-synd4 was used to activate synd 4 and its downstream signaling as described previously (13). Real-time PCR showed that synd 4 expression was increased in cardiomyocytes (Supplementary Figure 2). Ad-Larcz was used as control. Insulin-like growth factor-1 (IGF-1) and isoprenaline (ISO) were added to Ad-Lacz-transfected cells to induce typical physiological and pathological cell hypertrophy, respectively. Overexpression of synd4 nearly doubled the cell size. The extent of sarcoma organization was increased by about 1.5 -fold as measured by $\left({ }^{35} \mathrm{~S}\right)$ methionine incorporation. Similar results of cell size and protein synthesis were observed in both IGF-1 and ISO-treated cardiomyocytes (Figures 3A,B). Tunnel kit was used to assess cell apoptosis in response to these stimulations. ISO-treated cardiomyocytes appeared as increased tunel-positive cells. Neither IGF-1 nor synd 4 overexpression increased cardiomyocyte apoptosis, compared with the control group (Figure 3C).

Next, we examined the molecular phenotypes in synd4-overexpressed cardiomyocytes. The pathological hypertrophy was associated with genetic 
A
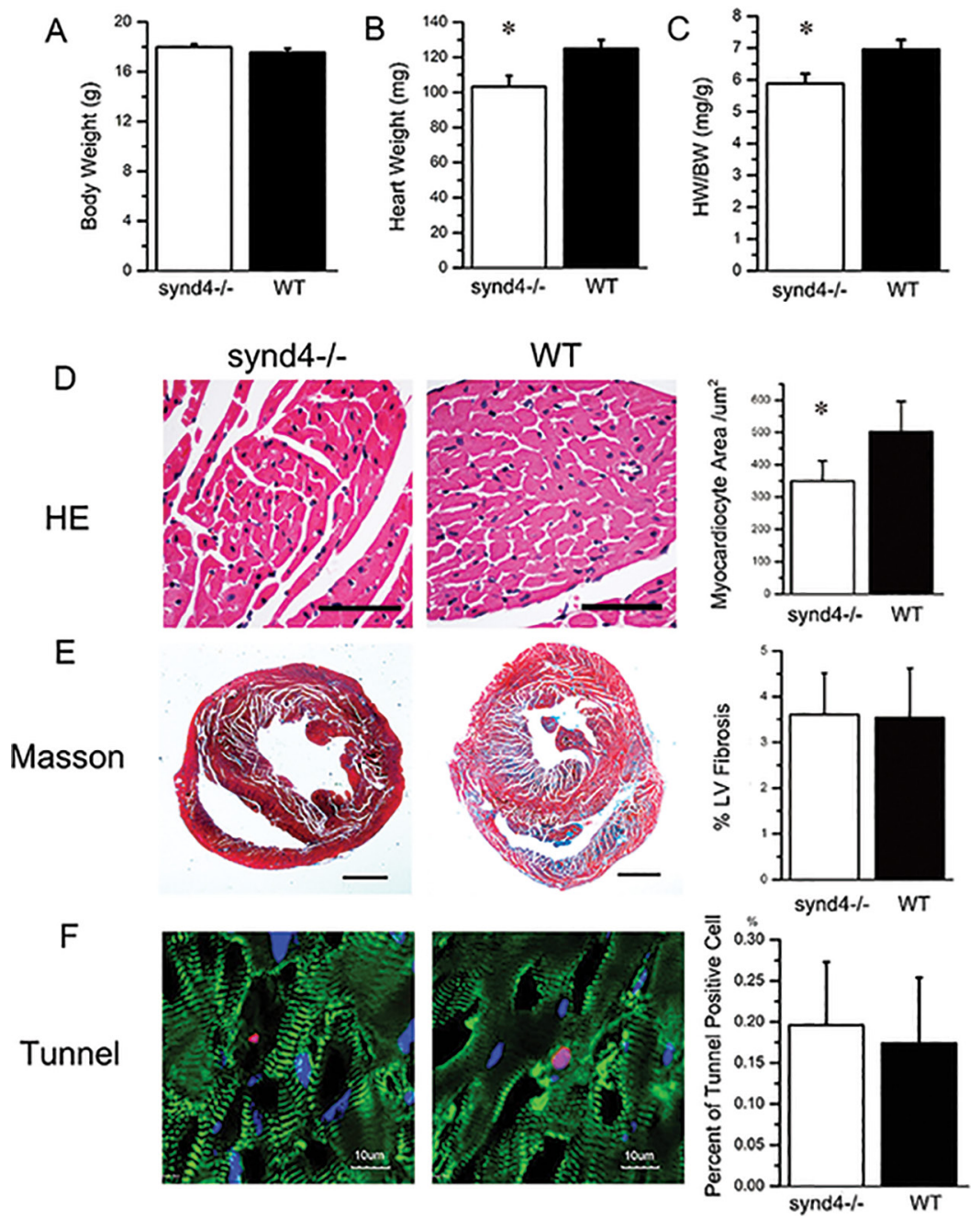

Figure 2. Exercise-induced cardiac hypertrophy was blunted in the synd $4^{-/-}$mice. (A to $C$ ): Histology analysis was used to detect body weight (BW), heart weight (HW) and HW/BW in synd $4^{-/-}$and WT mice after exercise. (B) The HW and (C) HW/BW were increased in WT mice compared with synd $4^{-/-}$mice after exercise. ${ }^{*} P<0.05$, synd $4^{-/-}$mice versus WT mice; $N=6$. (D) Histology analysis showed enlargement of cardiomyocytes in WT mice after exercise. ${ }^{*} P<0.05$, synd $4^{-/-}$mice versus WT mice; $\mathrm{N}=6$; scale bar $=100 \mu \mathrm{m}$. (E) Masson stain showed no difference of Masson-positive fibrosis in synd $4^{-/}$and WT mice after exercise. ${ }^{*} P<0.05$, synd $4^{-/-}$mice versus WT mice; $N=6$; scale bar $=1 \mathrm{~mm}$. (F) Tunel analysis showed no difference of tunel-positive apoptosis cells in synd $4^{-/}$and WT mice after exercise. ${ }^{*} P<0.05$, synd $4^{-/-}$mice versus WT mice; $\mathrm{N}=6$; scale bar $=10 \mu \mathrm{m}$.

reprogramming, the re-expression of a battery of fetal genes and the downregulation of multiple adult genes, which was not reported in physiological hypertrophy (22). We used real-time PCR to assess fetal and adult gene expression. We observed that three fetal genes, atrial natriuretic factor $(A N F), \beta$-myosin heavy chain
$(\beta-M H C)$ and $\alpha$-skeletal actin were upregulated in ISO-treated cell and not altered in synd4-overexpressed and IGF-1-treated cells. By contrast, synd4 overexpression and IGF-1 treatment could significantly induce expression of $\alpha$-myosin heavy chain $(\alpha-M H C)$, an adult gene (Figure 4A). Meanwhile, in energy metabolism, pathological hypertrophy is characterized by a switch from fatty acid utilization to glycolysis. In our study, the two lipid metabolism-related genes, peroxisome proliferator-activated receptor $\alpha(P P A R \alpha)$ and muscle carnitine palmitoyltransferase-1 (MCPT-1), were upregulated in synd4-overexpressed and IGF-1-treated cells, but not in ISO-treated cell. In addition, the expression of medium-chain acyl-CoA dehydrogenase $(M C A D)$, which is also involved in lipid metabolism, was slightly increased in synd4-overexpressed and IGF-1-treated cells (Figure 4B). Glucose metabolism was the major source of energy in pathological hypertrophy. In our study, we observed that glucose metabolism-related genes, lactate dehydrogenase $(L D H)$, hypoxia-inducible factors-1 $\alpha(H I F-1 \alpha)$, glucose transporter 1 (GLUT1), hexokinase 2 and pyruvate dehydrogenase lipoamide kinase isozyme 1 (PDK1) were upregulated in ISO-treated cells, but not in cells treated with IGF-1 or affected by synd 4 overexpression (Figure 4C). These results indicated the fetal gene reprogramming and glucose metabolism-related gene expression were not occurring in synd4-overexpressed cardiomyocytes.

In addition, we used Western blot to assess the protein level of PPAR $\alpha$ and ANF. The results were similar to those obtained in real-time PCR analysis. The protein level of PPAR $\alpha$ was increased in cells with synd4 overexpression and IGF-1 treatment. Also, the protein level of ANF was increased in ISO-treated cells. Taken together, these findings indicated synd4-induced hypertrophy was associated with molecular alternation observed in physiological rather than pathological hypertrophy.

\section{Synd4 Initiated Akt Signaling to Induce Cardiomyocyte Hypertrophy}

In a previous study, the calcineurinNFAT pathway was reported to be involved in synd4-related cardiac pathological hypertrophy $(14,15)$. Here, we assessed calcineurin-NFAT activation 


\section{A}
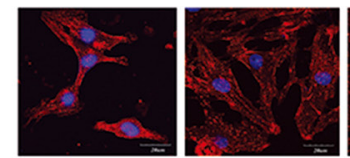

Ad-Lacz

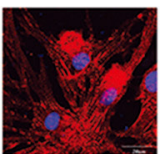

Ad-synd4

Ad-Lacz+IGF-1 Ad-Lacz+ISO
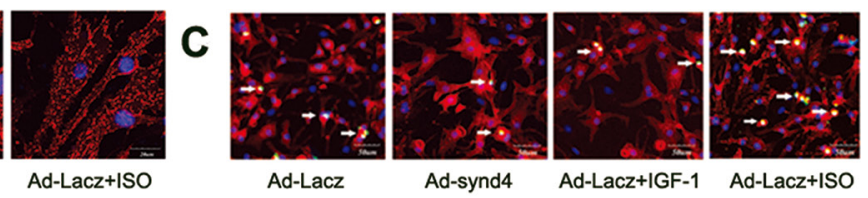

Ad-synd4

Ad-Lacz+IGF- 1

Ad-Lacz+ISO

Ad-lacz,

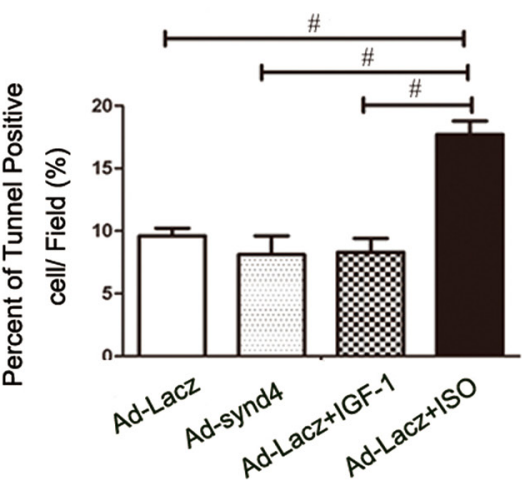

B
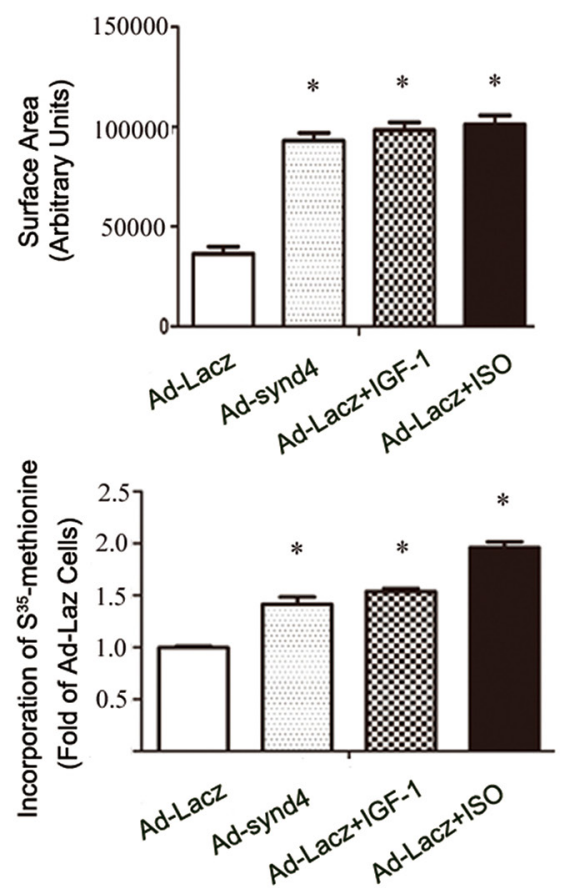

Figure 3. Synd4 overexpression induced cultured cardiomyocyte hypertrophy. Ad-synd4 was used to activate synd4. Ad-Lacz was used as control. IGF-1 (10 nmol/L) and ISO $(10 \mu \mathrm{m})$ were added to Ad-Lacz transfected cells to induce typical physiological and pathological cell hypertrophy respectively. (A) Immunofluorescence assay showed increased cell area in synd4-overexpressed, IGF-1-treated and ISO-treated cardiomyocytes. ${ }^{*} P<0.05$, Ad-synd4 versus Ad-Lacz, Ad-Lacz + IGF-1 versus Ad-Lacz, Ad-Lacz + ISO versus Ad-Lacz; scale bar $=20 \mu \mathrm{m} ; \mathrm{N}=3$ experiments, ANOVA. (B) $\left({ }^{35}\right.$ S)methionine incorporation assay showed increased protein production in synd4-overexpressed, IGF-1-treated and ISO-treated cardiomyocytes. ${ }^{*} P<0.05$, Ad-synd4 versus Ad-Lacz, Ad-Lacz + IGF-1 versus Ad-Lacz, Ad-Lacz + ISO versus Ad-Lacz; N = 3 experiments, ANOVA. (C) Tunel assay showed increase in tunel-positive cells in ISOtreated cardiomyocytes. ${ }^{*} P<0.05$, Ad-synd4 versus Ad-Lacz + ISO, Ad-Lacz + IGF-1 versus Ad-Lacz + ISO, Ad-Lac versus Ad-Lacz + ISO; scale bar $=50 \mu \mathrm{m} ; \mathrm{N}=3$ experiments, ANOVA.

in exercise mice. The calcineurin level was not changed between exercise and sedentary WT mice. After exercise training, no difference of the calcineurin level in myocardium was observed between synd $4^{-/-}$ and WT mice (Figure 5A). Meanwhile, the level of NFAT in cytoplasm and nucleus were not changed after exercise (Figures 5B,C). There results indicated that the calcineurin-NFAT pathway did not take part in synd4-dependent cardiac hypertrophy.

PI3K/Akt signaling was identified as the central kinase in cardiac physiological hypertrophy $(5,18)$. The level of Akt phosphorylation on serine 473 (S473) and threonine 308 (T308) were reported to be critical for Akt activation (23). We observed significantly decreased S473 and T308 Akt phosphorylation, in parallel with blunted cardiac physiological hypertrophy in synd $4^{-/-}$mice compared with WT mice after undergoing 4 wks of exercise (Figure 5D). In cardiomyocytes, synd4 overexpression could induce enlargement of cardiomyocytes and A6370, an Akt inhibitor, could significantly prevent synd4-induced cardiomyocyte hypertrophy (Figure 5E). These data indicate that the Akt pathway was required in synd4-dependent cardiac hypertrophy. Next, we used calphostin C to block PKC. We observed that calphostin $C$ could significantly prevent synd4-induced cardiomyocyte enlargement (Figure 5E). Western blot showed that overexpressed synd 4 could induce Akt phosphorylation in S473 and T308 on a significant level, and calphostin C could decrease synd4-induced phosphorylation of Akt at S473 and T308, in addition to its effect on cell hypertrophy (Figure 5F). These results indicated synd 4 could induce cardiomyocyte hypertrophy via PKC inducing Akt phosphorylation. 
A
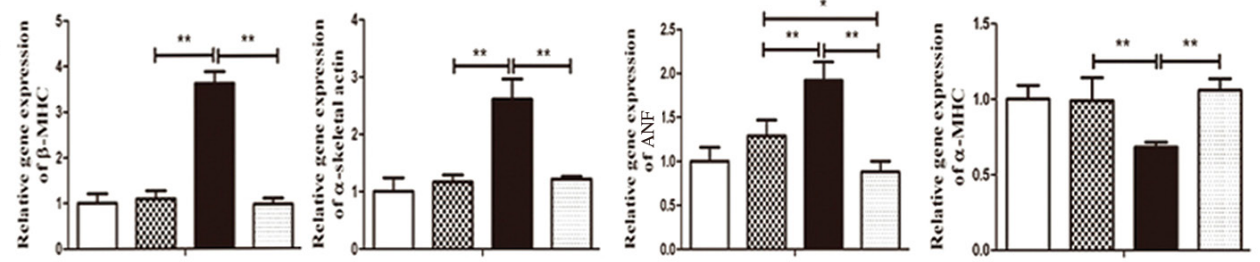

B
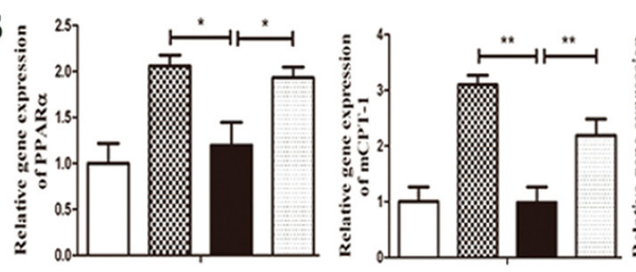

C
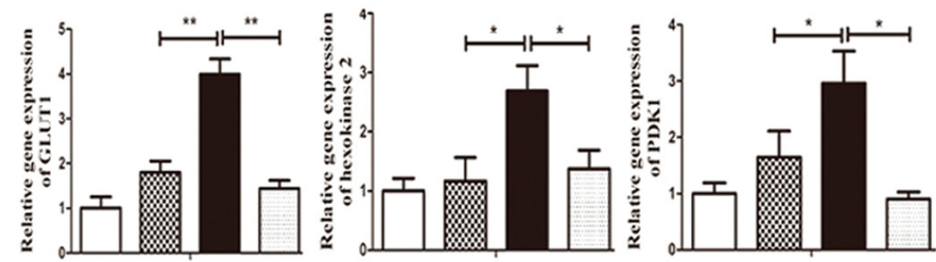

D
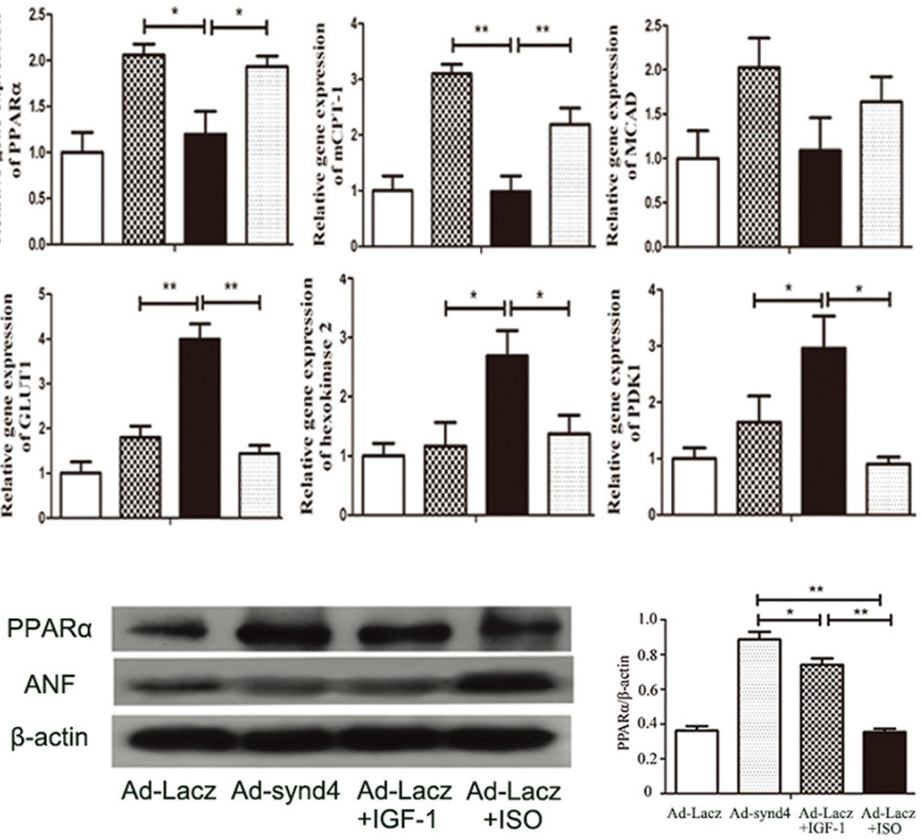

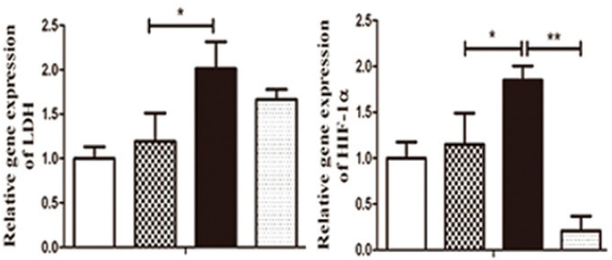

Figure 4. Synd4-induced cardiomyocytes hypertrophy was associated with distinct molecular phenotypes in physiological hypertrophy. Ad-synd 4 was used to activate synd4. Ad-Lacz was used as control. IGF-1 (10 nmol/L) and ISO (10 $\mu \mathrm{m})$ were added to Ad-Lacz-transfected cells to induce typical physiological and pathological cell hypertrophy respectively. Real-time PCR was used to assess gene expression in hypertrophic cardiomyocytes. (A) Fetal gene ( $\beta$-MHC, ANP and $\alpha$-skeletal actin) reprogramming was observed in ISO-treated, but not synd4-overexpressed, IGF-1-treated cardiomyocytes. ${ }^{*} P<0.05$, ${ }^{* *} P<0.01$, Ad-synd4 versus Ad-Lacz + ISO, Ad-Lacz + IGF-1 versus Ad-Lacz + ISO; $\mathrm{N}=3$ experiments, ANOVA; (B) The lipid metabolism-related genes (PPAR $\alpha, \mathrm{mCPT}-1$ and MCAD) were upregulated in synd4-overexpressed and IGF-1-treated cardiomyocytes. ${ }^{*} P<0.05,{ }^{* *} P<0.01$, Ad-synd4 versus Ad-Lacz + ISO, Ad-Lacz + IGF-1 versus Ad-Lacz + ISO; N = 3 experiments, ANOVA; (C) By contrast, the glucose metabolism-related genes (GLUT1, hexokinase 2, PDKI, LDH, HIF-1 $\alpha$ ) upregulated in ISO-treated cardiomyocytes. ${ }^{*} P<0.05,{ }^{* *} P<0.01$, Ad-synd4 versus Ad-Lacz + ISO, Ad-Lacz + IGF-1 versus Ad-Lacz + ISO; $N=3$ experiments, ANOVA; (D) Western blot showed increases of PPAR $\alpha$ expression in synd4-overexpressed and IGF-1-treated cardiomyocytes, and increases of ANF in ISO-treated cardiomyocytes. Data are shown as a ratio to $\beta$-actin. ${ }^{*} P<0.05,{ }^{* *} P<0.01$, Ad-synd4 versus Ad-Lacz + ISO, Ad-Lacz + IGF-1 versus Ad-Lacz + ISO, Ad-synd4 versus Ad-Lacz + IGF-1, Ad-Lacz versus Ad-Lacz + ISO; $\mathrm{N}=3$ experiments, ANOVA; ANP: atrial natriuretic peptide.

\section{DISCUSSION}

In this study, we have focused on the role of synd4 in physiological cardiac hypertrophy. We observed that synd 4 was upregulated in the myocardium of exercise-trained rats. The exercise-induced cardiac adaptation was blunted when synd 4 was knocked out. In cardiomyocytes, synd 4 overexpression could induce cell hypertrophy. The hypertrophic phenotypes in synd4-verexpressed cardiomyocytes were similar to those induced by IGF-1. In the end, we showed Akt signaling took part in synd4-induced cardiomyocytes hypertrophy.

Cardiac growth or hypertrophy can be broadly classified as either physiological or pathological. Physiological heart growth includes normal postnatal growth, pregnancy-induced growth, and exercise-induced cardiac hypertrophy.
By contrast, pathological growth occurs in response to chronic pressure or volume overload in a disease setting (4). Despite comparable increases in heart size, pathological and physiological hypertrophy is associated with distinct phenotypes in myocardium and cardiomyocytes. Compared with physiological hypertrophy, pathological hypertrophy is associated with cell death, fibrosis (24) and insufficient 
A
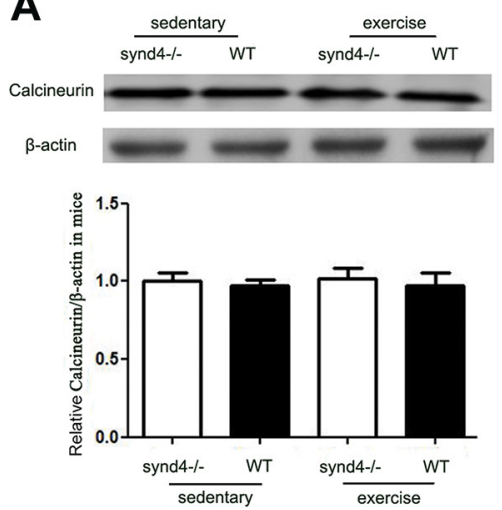

B

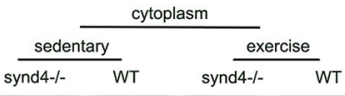
synd4-l- WT synd4-l- WT
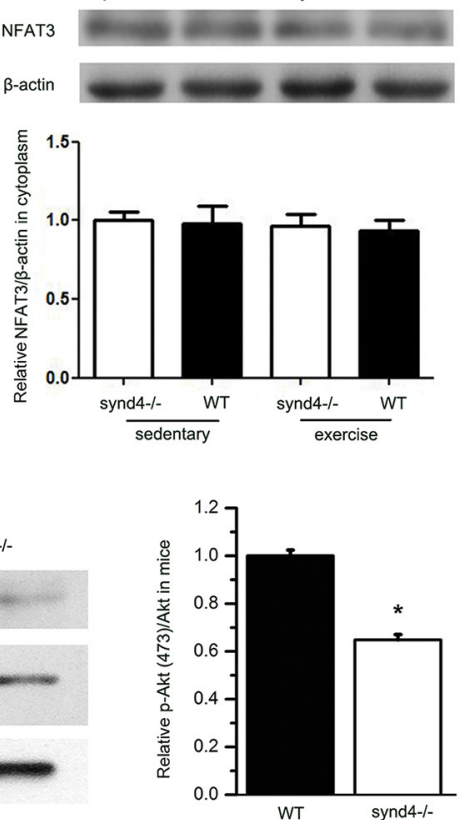
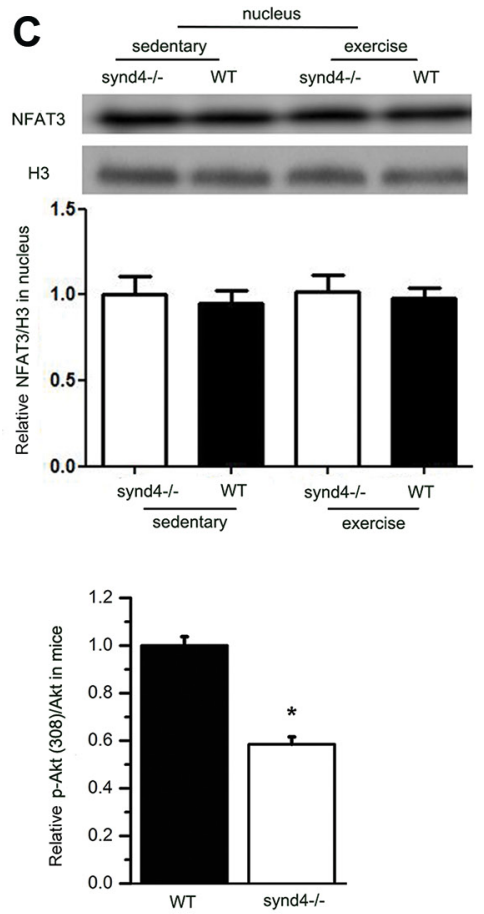

\section{E}

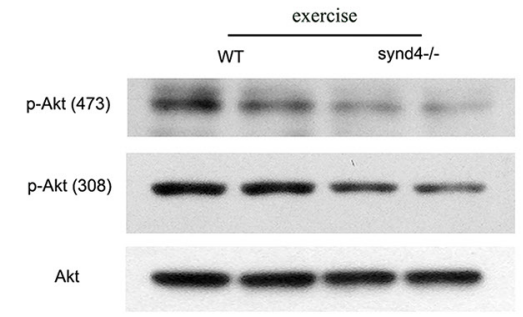

$\frac{\text { exercise }}{\text { WT }} \quad$ synd4 $-I$

D

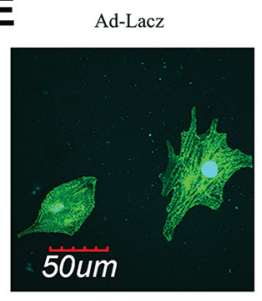

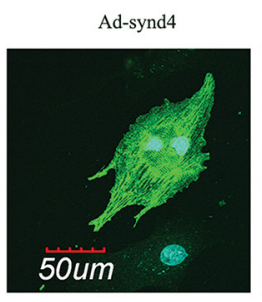

Ad-synd4+A6730

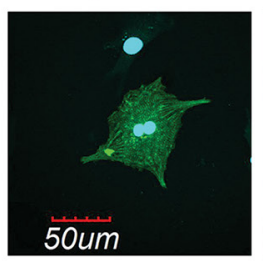

Ad-synd4+Calphostin C

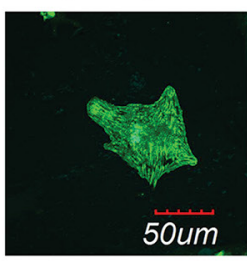

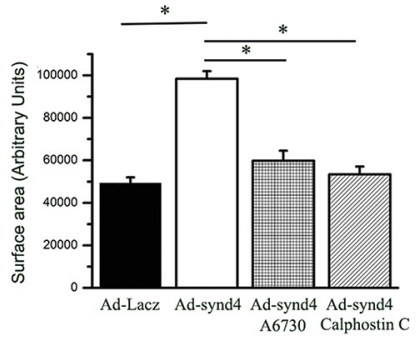
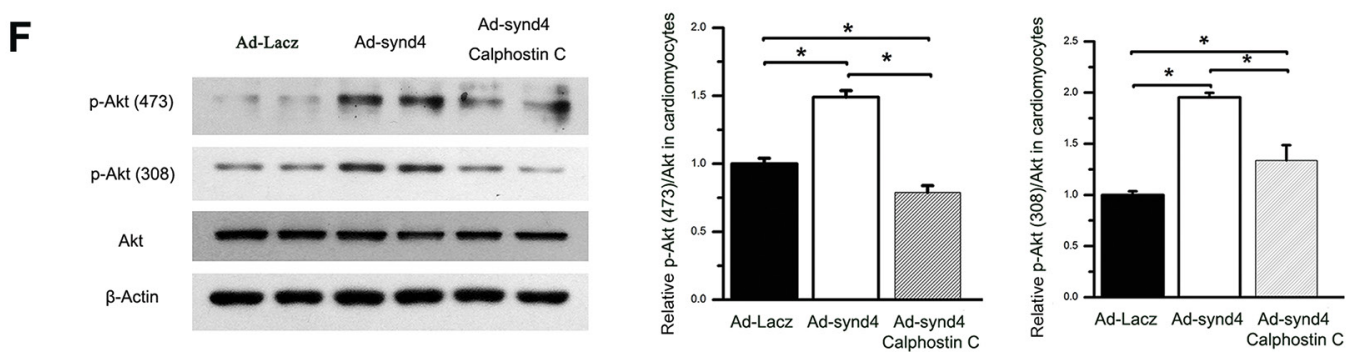

Figure 5. Synd4 induced cardiomyocytes hypertrophy via Akt phosphorylation. (A-C) Western blot showed no changes of (A) calcineurin and (B and C) its downstream targets, NFAT3 in myocardium between WT and synd $4^{-/-}$mice, as well as between sedentary and exercise mice. Data are shown as a ratio to $\beta$-actin or histone $\mathrm{H} 3$ and are expressed as a fold-increase when compared with sedentary synd $4^{-/-}$mice. $\mathrm{N}=6$, ANOVA; (D) Western blot showed decreased $\mathrm{S} 473$ and T308 Akt phosphorylation in synd $4^{-/-}$mice after exercise. Data are shown as a ratio to total Akt and are expressed as a fold-increase when compared with WT mice. ${ }^{*} P<0.05$, synd4 ${ }^{-1-}$ mice versus WT mice, $\mathrm{N}=6$, ANOVA; Ad-synd4 was used to activate synd4 in cultured cardiomyocyte. (E) Immunofluorescence assay showed synd4 overexpression could induce cardiomyocyte enlargement. A6730 (Akt inhibitor, $5 \mu$ mol/L) and calphostin C (PKC inhibitor, $100 \mathrm{nmol} / \mathrm{L}$ ) could attenuate synd4 depended cardiomyocyte enlargement. ${ }^{*} \mathrm{P}<0.05$, Ad-Lacz versus Ad-synd4, Ad-synd4 + A6730 versus Ad-synd4, Ad-synd4 + calphostin C versus Ad-synd4; $N=3$ experiments, ANOVA; (F) Western blot showed synd4 overexpression could increase Akt phosphorylation in $\$ 473$ and T308. Calphostin C could blunt synd4-induced Akt phosphorylation. Data are shown as a ratio to total Akt and are expressed as a fold-increase when compared with Ad-Lacz groups. ${ }^{*} P<0.05$, Ad-Lacz versus Ad-synd4, Ad-synd4 + calphostin C versus Ad-synd4; $N=3$ experiments, ANOVA. 
angiogenesis (25) in myocardium. Meanwhile the switching energy substrates from fatty acid to glucose (26) and fetal genes reprogramming (22) were observed in pathological hypertrophic cardiomyocytes. In this study, cardiac hypertrophy was induced by high intense, aerobic interval swimming training and blunted in synd $4^{-/-}$mice. No increased cell apoptosis and fibrosis was observed in WT and synd $4^{-/-}$mice after swimming. In cardiomyocytes, cell enlargement without apoptosis was observed in synd4-overexpressed cells. The genes affecting fatty metabolism rather than glucose metabolism were upregulated, indicating that fatty acid oxidation was still the main source of energy generation in cardiomyocytes that expressed synd4. No fetal gene expression was observed under synd 4 overexpression. These results suggested that cardiomyocytes with synd4-induced hypotrophy have typical features of physiological hypertrophy. Hence, through animal and cell studies, we demonstrated that synd4 was involved in physiological cardiac hypertrophy.

Our study focused firstly on the effect of synd4 on exercise-induced physiological cardiac hypertrophy and added some new insight into the relationship between synd 4 and cardiac hypertrophy. The feature and underlying molecular signaling in synd4-induced hypertrophy were different and dependent on the heart's physiological or pathological condition. In the aortic constriction animal model, synd 4 was observed to initiate cardiomyocyte hypertrophy (15) and tissue fibrosis (27). In our swimming animal model, physiological cardiac hypertrophy without fibrosis was observed. No data indicated fibroblasts were activated in the physiological myocardium. Hence, we focused only on the synd4-dependent pathway in cardiomyocytes. In a previous study, the NFAT pathway was regulated by synd 4 and modified in pathological processes in cardiomyocytes. Here, we demonstrated that Akt rather than calcineurin-NFAT took part in synd4-dependent physiological cardiac hypertrophy. The phosphorylation of Akt was blunted in synd $4^{-/-}$mice under swimming training, in addition to the attenuation of physiological cardiac hypertrophy. The PI3K/Akt pathway regulated numerous cellular functions, including proliferation, migration and adhesion $(28,29)$. Consequently, the $\mathrm{PI} 3 \mathrm{~K} /$ Akt pathway is a key regulator of physiological cardiac hypertrophy $(18,30)$. The mice without Akt1 were resistant to swimming training-induced cardiac hypertrophy. Therefore, we suggested that under physiological stimulations synd 4 initiated Akt to regulate cardiac hypertrophy.

The activation of Akt needed simultaneous phosphorylation of S473 and T308. Synd 4 could regulate Akt phosphorylation on $\$ 473$ by regulating the intracellular localization of mTORC2 via PKC $\alpha$ (17). On the other hand, synd4-dependent activation of PKC $\alpha$ was required for PAK-mediated recruitment of PDK1 and phosphorylation of Akt T308 (16). We observed that both S473 and T308 phosphorylation of Akt was blunted in synd $4^{-/-}$ mice under the swimming training. We further demonstrated that synd 4 induced Akt phosphorylation on S473 and T308 in the PKC-dependent manner in vitro. Hence, we indicated that synd 4 could activate Akt via PKC in the process of physiological cardiomyocyte hypertrophy.

IGF-1 was a classical growth factor in initiating physiological cardiac hypertrophy (31). Several previous works indicated synd 4 could act as an IGF-1 receptor. Rong et al. reported that IGF-1-induced Akt phosphorylation was significantly decreased in synd $4^{-/-}$ cells (16). Other reports demonstrated that synd 4 binds tissue transglutaminase (TG2), which cross-linked various extracellular matrix (ECM) proteins and soluble growth factors by noncovalent interactions, including IGF-1 (32-34). Synd 4 may modulate IGF-1 downstream signaling via its interaction with a TG2-IGF-1 complex. In this study, we observed that synd 4 and its signaling could induce physiological cardiac hypertrophy. Whether IGF-1 was one of the important ligands to initiate synd4-dependent physiological cardiac hypertrophy needs to be elucidated in future.

It is worth noting that synd4 interacted with not only IGF-1 but also with other ligands nonspecifically. These ligands include growth factors and components of ECM. The recognition that alterations in ECM contributed to cardiac hypertrophy has resulted in a growing interest in recent years (35). Whether the interaction between ECM and cardiomyocytes is involved in physiological cardiac hypertrophy needs further elucidation. Synd4 may act as the nonspecific but fundamental receptor to transduce signaling concerning physiological hypertrophy of cardiomyocytes from ECM to cytoplasm.

A necessary methodological limitation in our study was the global knockout model; unfortunately, this was unavoidable since synd 4 is synthesized in many different cell types including smooth muscle cells, endothelial cells, cardiomyocytes and fibroblasts. Therefore, we cannot exclude potential systemic, indirect, non-cardiac effects upon hypertrophy. However, in vitro studies, which reduce the variables to a minimum, demonstrate the direct effects of synd 4 on cardiomyocytes (irrespective of the cellular source of synd 4 in vivo and potential systemic effects). Another methodological limitation was that we used Ad-synd4, but not ligands, to activate a synd 4 dependent pathway in cardiomyocytes. A previous study reported that expression of synd 4 could induce homo-oligomers with itself, resulting in activation of the C-terminal tail of synd 4 and initiating its downstream signaling (36). Because synd 4 binds several ligands nonspecifically on the cell surface, there was no specific ligand that could be used to activate synd4. Hence we used Ad-synd4 in our cell study.

\section{CONCLUSION}

This study showed synd 4 expression was increased in physiological hypertrophic myocardium. When synd 4 
was knocked out, the physiological cardiac hypertrophy was blunted. In cardiomyocytes, synd 4 overexpression induced cell enlargement and typical molecular alternation of physiological hypertrophy. In addition, Akt signaling was a key responder under synd4 in moderating physiological cardiac hypertrophy. In a previous study, other authors reported synd4 took part in pathological cardiac hypertrophy. The result of our current study added some new insight into the relationship between synd 4 and cardiac hypertrophy. We demonstrated that synd 4 was also key in initiating physiological cardiac hypertrophy. The phenotype of synd4-induced hypertrophy was different and depended on the heart's physiological or pathological condition.

\section{ACKNOWLEDGMENTS}

This work was supported by grants from the Natural Science Foundation of China (81200148, 81270281, 81470371, 81000055), the Jiangsu Provincial Special Program of Medical Science (BL2012014), the State Key Laboratory of Pharmaceutical Biotechnology (KF-GN-200901), the Peak of Six Personnel in Jiangsu Province (2013-WSN-008), the Funds for Distinguished Young Scientists in Nanjing (JQX13006) and the Key Program of the Science Foundation in Nanjing (ZKX13023).

\section{DISCLOSURE}

The authors declare that they have no competing interests as defined by Molecular Medicine, or other interests that might be perceived to influence the results and discussion reported in this paper.

\section{REFERENCES}

1. Kokkinos P, Myers J. (2010) Exercise and physical activity: clinical outcomes and applications. Circulation. 122:1637-48.

2. Manson JE, et al. (2002) Walking compared with vigorous exercise for the prevention of cardiovascular events in women. N. Engl. J. Med. 347:716-25.

3. Wisloff U, et al. (2007) Superior cardiovascular effect of aerobic interval training versus moderate continuous training in heart failure patients: a randomized study. Circulation. 115:3086-94.

4. Bernardo BC, Weeks KL, Pretorius L, McMullen JR. (2010) Molecular distinction between physiological and pathological cardiac hypertrophy: experimental findings and therapeutic strategies. Pharmacol. Ther. 128:191-227.

5. McMullen JR, et al. (2003) Phosphoinositide 3-kinase (p110alpha) plays a critical role for the induction of physiological, but not pathological, cardiac hypertrophy. Proc. Natl. Acad. Sci. U. S. A. 100:12355-60.

6. Bourajjaj M, et al. (2008) NFATc2 is a necessary mediator of calcineurin-dependent cardiac hypertrophy and heart failure. J. Biol. Chem. 283:22295-303.

7. Shende $\mathrm{P}$, et al. (2011) Cardiac raptor ablation impairs adaptive hypertrophy, alters metabolic gene expression, and causes heart failure in mice. Circulation. 123:1073-82.

8. Nakayama H, et al. (2009) alpha1G-dependent T-type Ca2+ current antagonizes cardiac hypertrophy through a NOS3-dependent mechanism in mice. J. Clin. Invest. 119:3787-96.

9. Couchman JR. (2010) Transmembrane signaling proteoglycans. Annu. Rev. Cell Dev. Biol. 26:89-114.

10. VanWinkle WB, et al. (2002) Couchman JR. Localization of the transmembrane proteoglycan syndecan- 4 and its regulatory kinases in costameres of rat cardiomyocytes: a deconvolution microscopic study. Anat. Rec. 268:38-46.

11. Keum E, et al. (2004) Syndecan-4 regulates localization, activity and stability of protein kinase C-alpha. Biochem. J. 378:1007-14.

12. Jalili T, Takeishi Y, Walsh RA. (1999) Signal transduction during cardiac hypertrophy: the role of G alpha q, PLC beta I, and PKC. Cardiovasc. Res. 44:5-9.

13. Xie J, et al. (2012) Syndecan-4 over-expression preserves cardiac function in a rat model of myocardial infarction. J. Mol. Cell. Cardiol. 53:250-8.

14. Echtermeyer F, et al. (2011) Syndecan-4 signalling inhibits apoptosis and controls NFAT activity during myocardial damage and remodelling. Cardiovasc. Res. 92:123-31.

15. Finsen AV, et al. (2011) Syndecan-4 is essential for development of concentric myocardial hypertrophy via stretch-induced activation of the calcineurin-NFAT pathway. PLoS One. 6:e28302.

16. Ju R, Simons M. (2013) Syndecan 4 regulation of PDK1-dependent Akt activation. Cell Signal. 25:101-5.

17. Partovian C, Ju R, Zhuang ZW, Martin KA, Simons M. (2008) Syndecan-4 regulates subcellular localization of mTOR complex2 and Akt activation in a PKCalpha-dependent manner in endothelial cells. Mol. Cell. 32:140-9.

18. DeBosch B, et al. (2006) Akt1 is required for physiological cardiac growth. Circulation. 113:2097-104.

19. Taniike M, et al. (2008) Apoptosis signal-regulating kinase 1/p38 signaling pathway negatively regulates physiological hypertrophy. Circulation. 117:545-52.

20. Li Q, et al. (2014) Overexpression of microRNA-99a attenuates heart remodelling and improves cardiac performance after myocardial infarction. J. Cell. Mol. Med. 18:919-28.
21. Matsui Y, et al. (2011) Syndecan-4 prevents cardiac rupture and dysfunction after myocardial infarction. Circ. Res. 108:1328-39.

22. Iemitsu M, et al. (2001) Physiological and pathological cardiac hypertrophy induce different molecular phenotypes in the rat. Am. J. Physiol. Regul. Integr. Comp. Physiol. 281:R2029-36.

23. Kemi OJ, et al. (2008) Activation or inactivation of cardiac Akt/mTOR signaling diverges physiological from pathological hypertrophy. J. Cell. Physiol. 214:316-21.

24. McMullen JR, Jennings GL. (2007) Differences between pathological and physiological cardiac hypertrophy: novel therapeutic strategies to treat heart failure. Clin. Exp. Pharmacol. Physiol. 34:255-62.

25. Oka T, Akazawa H, Naito AT, Komuro I. (2014) Angiogenesis and cardiac hypertrophy: maintenance of cardiac function and causative roles in heart failure. Circ. Res. 114:565-71.

26. Davila-Roman VG, et al. (2002) Altered myocardial fatty acid and glucose metabolism in idiopathic dilated cardiomyopathy. J. Am. Coll. Cardiol. 40:271-7.

27. Herum KM, et al. (2013) Syndecan-4 signaling via NFAT regulates extracellular matrix production and cardiac myofibroblast differentiation in response to mechanical stress. J. Mol. Cell. Cardiol. 54:73-81.

28. Kawasaki K, et al. (2003) Activation of the phosphatidylinositol 3-kinase/protein kinase Akt pathway mediates nitric oxide-induced endothelial cell migration and angiogenesis. Mol. Cell. Biol. 23:5726-37.

29. Shiojima I, Walsh K. (2002) Role of Akt signaling in vascular homeostasis and angiogenesis. Circ. Res. 90:1243-50.

30. Chaanine AH, Hajjar RJ. (2011) AKT signalling in the failing heart. Eur. J. Heart Fail. 13:825-9.

31. Ikeda $H$, et al. (2009) Interaction of myocardial insulin receptor and IGF receptor signaling in exercise-induced cardiac hypertrophy. J. Mol. Cell. Cardiol. 47:664-75.

32. Belkin AM. (2011) Extracellular TG2: emerging functions and regulation. FEBS J. 278:4704-16.

33. Dardik R, Inbal A. (2006) Complex formation between tissue transglutaminase II (tTG) and vascular endothelial growth factor receptor 2 (VEGFR-2): proposed mechanism for modulation of endothelial cell response to VEGF. Exp. Cell Res. 312:2973-82.

34. Zemskov EA, et al. (2009) Regulation of platelet-derived growth factor receptor function by integrin-associated cell surface transglutaminase. J. Biol. Chem. 284:16693-703.

35. Fujiu K, Nagai R. (2014) Fibroblast-mediated pathways in cardiac hypertrophy. J. Mol. Cell. Cardiol. 70:64-73.

36. Couchman JR. (2003) Syndecans: proteoglycan regulators of cell-surface microdomains? Nat. Rev. Mol. Cell. Biol. 4:926-37.

Cite this article as: Xie J, et al. (2016) Syndecan-4 signaling is required for exercise-induced cardiac hypertrophy. Mol. Med. 22:192-201. 